\title{
Characterization of Immunophenotypic Aberrancies in Adult and Childhood Acute Lymphoblastic Leukemia: Lessons from Regional Variation
}

\author{
Mitra Sadat Rezaei ${ }^{1,2,3 *(D)}$, Najmeh Esfandiari ${ }^{3}$, Sandra Refoua $^{2}$, Masoud Shamaei ${ }^{4}$ \\ 1. Virology Research Center, National Research Institute of Tuberculosis and Lung Diseases, Shahid Beheshti University of Medical Sciences, \\ Tehran, Iran \\ 2. High Institute for Research and Education in Blood Transfusion Medicine, Iranian Blood Transfusion Organization, Tehran, Iran \\ 3. Department of Pathology, School of Medicine, Shahid Beheshti University of Medical Sciences, Tehran, Iran \\ 4. Clinical Tuberculosis and Epidemiology Research Center, National Research Institute of Tuberculosis, Tehran, Iran
}

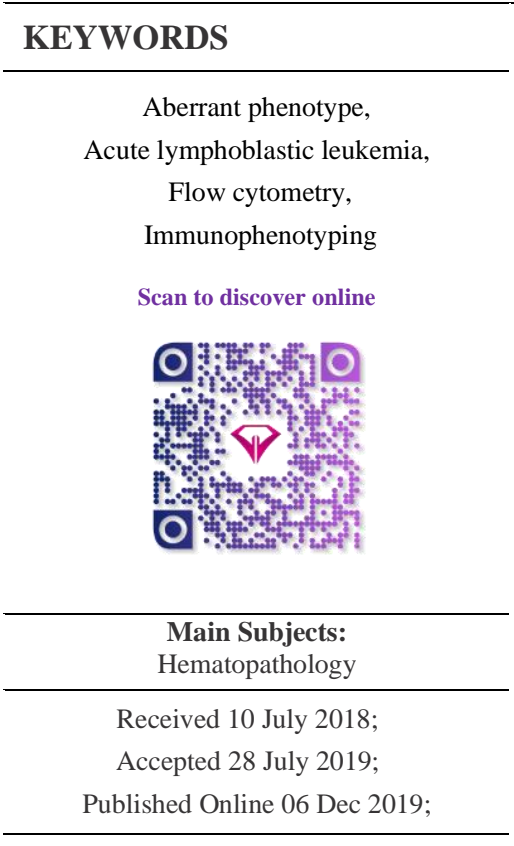

10.30699/IJP.2019.93974.1926

PMCID: PMC6995678

PMID: 32095142

\begin{abstract}
Background \& Objective: Although the antigen expression patterns of acute lymphoblastic leukemia (ALL) are well known, this study attempted to evaluate commonly used immune markers for immunophenotyping of acute leukemia to set the minimum of necessary diagnostic panels by flow cytometry.
\end{abstract}

Methods: This study evaluated 89 patients referred from all over the country to the Iranian Blood Transfusion Organization (IBTO) in Tehran from 2013 to 2015. We compared the immunophenotype patterns of childhood and adult ALLs including 69(77.5\%) B-cell lymphoblastic lymphoma (B-LBL), 2(2.2\%) Burkitt's lymphoma (BL), and 18(20.2\%) T-cell lymphoblastic lymphoma (T-LBL) cases using flowcytometry with broad antibody panel.

Results: CD19 and CD79a were the most frequent markers for B-LBL while CD7 was the most sensitive marker in T-LBL; the frequency of CD7, CD3, and CD5 antigens were $100 \%, 38.9 \%$, and $88.9 \%$, respectively. TdT+/CD34+ was significantly higher in adult B-LBLs than children, which indicates blast cells are more immature in adults. In addition, CD10 and cCD79a were significantly higher in children with B-LBL like as CD5 and CD8 in children with T-LBL. Aberrant phenotypes including CD13, CD33, CD7, and CD117 were found in $7(10.1 \%)$ cases of B-LBL. These phenotypes were CD117, HLA-DR, and CD33 in 7(38/9\%) cases of T-LBL. Expression of CD117 aberrant myeloid antigen was significantly more associated with T-LBL than with Blineage ALL.

Conclusion: Significant differences were observed in antigen-expression patterns between adult and childhood ALLs. Further studies are needed to correlate specific markers with recurrent cytogenetic abnormalities and prognosis with therapeutic response.

\section{Corresponding Information:}

Mitra Sadat Rezaei, Virology Research Center, National Research Institute of Tuberculosis and Lung Diseases, Shahid Beheshti University of Medical Sciences, Tehran, Iran Email: dr_mrezaie@yahoo.com

Copyright (C) 2020. This is an open-access article distributed under the terms of the Creative Commons Attribution- 4.0 International License which permit Share, copy and redistribution of the material in any medium or format or adapt, remix, transform, and build upon the material for any purpose, even commercially.

\section{Introduction}

Acute lymphoblastic leukemia (ALL) includes a group of proliferative disorders of the bone marrow and it is considered as one of the most common malignancies in children (1). Although the peak of prevalence is between the ages of 2 and 5 years, ALL affects both children and adults (2). This prevalence increases by age up to the second peak in the 80 years old (1). Cancer incidence is the third cause of death in Iran after coronary heart disease and car accidents (3) and the pediatric cancer is the sixth common malignancy according to different studies in Iran (4-6).

Although chromosomal analysis still plays an important role in cytogenetic studies, morphological identification of lymphoblast with light microscopy and immunophenotypic evaluation of commitment lineage and developmental stage with the flow cytometry are essential for detection (2).

Immunological characteristic of leukemic cells is extensively used by flow cytometry both with surface and cytoplasmic antigens, which are actually different in developmental stage and lineage of malignant cells (7). The identification of neoplastic cells by flow cytometry relies on the principle that the expression patterns of markers in normal and neoplastic cells are different. This includes acquisition/changes in intensity or loss of antigen expression which are not lineage specific (8). Today, we have appropriate antibody panel to study this lineage of the malignant cells by flow cytometry, but actually most of the individual cell differentiation antigens cannot identify specific lineage (9), so immunologic 
classification of all acute leukemia needs a panel of monoclonal antibodies (mAb), rather than investigation for single antigen. On the other hand, there is an overlap in expression for some of the markers throughout acute leukemia of different lineages, which necessitates to use several markers. For example, CD33 may be positive in precursor of B- or T-lymphoblastic leukemia (B-LBL and T- LBL); CD19 may be positive in a subset of AML-M2, and some of the pan-T antigens (CD2 and CD7) are often expressed in AML (10).

Although many studies have been done on ALL immunophenotyping, their results of flow cytometry panels are different by regions (11-14). This study, for the first time, evaluated aberrancies in ALL flow cytometry panels according to subtypes including B-LBL, T-LBL, and sex in both children and adults in Iran. The study tries to achieve better understanding of the diagnosis and classification of ALL through describing the characterization of immunophenotypic aberrancies using flow cytometry.

\section{Materials and Methods \\ Patient Selection}

The patients referred to the Iranian Blood Transfusion Organization (IBTO) in Tehran from all Iranian cities. The participants were examined in the flow cytometry section and entered the study. 89 blood samples were collected from childhood to adult in a time period between 2013 and 2015. The study protocol was reviewed, approved, and supervised by IBTO ethics committees.

\section{Flow Cytometry}

Before antibody staining, specimen processing was done by erythrocyte lysis with 5-volume ammonium chloride standard solution, and the complex incubated for 10-20 min according to lysis process. The samples were then washed two times with PBS solution (phosphatebuffered saline, $0.0455 \%$ sodium azide, and $0.1 \%$ bovine serum albumin) and resuspended in RPMI 1640 culture medium supplemented with $5 \%$ new borne calf serum (11). Finally, cell counting was done and 500,000 cells were washed and stained with antibodies.

Flow cytometry panels for T-LBL included CD1a, TdT, CD7, CD5, CD8, CD4, CCD3, CD3, and CD2, while the panels for B-LBL included CD22, CD20, CD19, CD34, CD79a, CCD22, and other antigens including HLA-DR, CD34, TdT, and CD45. In addition, CD13, CD33, CD14, CD117, CD64, and MPO antibodies were used to rule out AML. All antibodies were added according to manufacturer's protocols (Dako, Denmark) and then titration was done. Then, the specimens were incubated in dark room $\left(2-8^{\circ} \mathrm{C}\right)$ for $20 \mathrm{~min}$. After incubation, one wash with PBS was done and then resuspended in PBS. Flow cytometric analysis was performed using a Partec PAS flow cytometer (Partec $\mathrm{GmbH}$, Münster, Germany). Cell population was considered as positive for each particular antigen if it was more than $20 \%$ of the leukemic lymphoblast event stained beyond an appropriate isotype cutoff (set at $98 \%$ isotypic control staining). Acquisition and analysis of FCM data was performed using the FloMax program associated with the instrument.

\section{Data Analysis}

Immunophenotypic aberrancies in leukemic lymphoblast population was determined by deviation from normal pattern (15). The difference in frequency of antigens expression (categorical variable) between BLBL and T-LBL according to sex and age groups [children (under 14 years) and adults (over 14 years] was analyzed using Chi-square (Fisher exact test for expected frequency with or less than 5 percent). A P-value less than 0.05 was considered statistically significant.

\section{Results}

In this study, 89 patients including 54 males $(60.7 \%)$ and 35 females $(39.3 \%)$ were evaluated using the same methods. The mean age was 19.02 \pm 18.56 (Mean \pm SD). These patients had a wide age range of one year to 83 years old. Most of these patients $(62.9 \%)$ aged less than 14 years. Diagnosis was B-LBL for 69 patients $(77.5 \%)$, T-LBL for 18 patients (20.2\%), and mature B-LBL for 2 patients $(2.2 \%)$.

In the patients with B-ALL, 86.2\% TdT+, 79.4\% $\mathrm{CD} 10+, 1.47 \% \mathrm{CD} 117+$, and $60.9 \%$ were CD34+. Analysis of B-LBL precursor demonstrated $10.7 \%$ were TdT-/CD34-, 0\% TdT-/CD34+, 33/9 TdT+/CD34-, $55 / 3 \% \mathrm{TdT}+/ \mathrm{CD} 34+, 1.47 \% \mathrm{CD} 117+, 5.8 \% \mathrm{CD} 13+$, and $2.9 \%$ were $\mathrm{CD} 33+$.

None of B-LBL cases were MPO+. CD19 marker was positive in all B-LBL cases. CD20 expression was observed in $43.5 \%$ cases and CD22 expression in $25 \%$ cases, while $45.8 \%$ of cases were positive for CD22. In addition, CCD79a in $96.5 \%$ of cases was positive and HLADR in $98.6 \%$ cases (Table 1 ).

Aberrant B-LBL antigens were CD7, CD13, CD117 and CD 33. Immunophenotypic aberrancies in this disease include abnormal or a synchronized patterns of antigen expression for myeloid and lymphoid lineage (Table 2)

In T-LBL group CD4+/CD8+ patients were more in females than males, while CD4-/CD8- cases were more seen in the males. None of the women was CD10 positive, while $26.7 \%$ of T-LBL in males was positive for CD10. CD45 low in this group was seen more in females (Table 1). CD13, CD117, and HLADR were observed in males over 14 years old but CD10 was seen only in males under 14 years old (Table 3 ). In addition, expression of CD117 aberrant myeloid antigen was significantly more associated with T-LBL than with B-lineage ALL $(P=0.02)$ (Table 4). $11.1 \%$ of patients were CD4-/CD8+, and CD10 expression was seen in $22 / 2 \%$ of the patients. CD34+ was seen in $27 / 7 \%$, HLADR in $11 / 1 \%$, CD33 in $5.6 \%$, CD117 in $13 / 3 \%$, TdT+/CD34+ in $7.14 \%$, TdT$/ \mathrm{CD} 34-$ in $28.6 \%$,TdT-/CD34+ in $14.3 \%$, and TdT+/CD34- in $50 \%$ of T-LBL patients (Table 1). CD4 and CD1a markers were not expressed in adults. These results should be interpreted by considering limited numbers of cases in the T-LBL group. In addition, CD8 and CD5 antigen expression was higher in the group of children (Table 1).. 
Table 1. Patients characteristics and immunophenotypic profile of patients with ALL

\begin{tabular}{|c|c|c|c|c|c|c|c|c|c|c|c|c|c|c|}
\hline \multirow{2}{*}{ Variable } & \multirow{2}{*}{$\begin{array}{c}\text { B.ALL } \\
\text { No }(\%) \\
(n=69)\end{array}$} & \multirow{2}{*}{$\begin{array}{c}\text { T.ALL } \\
\text { No }(\%) \\
(n=18)\end{array}$} & & \multicolumn{2}{|c|}{ B.ALL No(\%) } & & \multicolumn{2}{|c|}{ T.ALL No(\%) } & \multicolumn{2}{|c|}{ B.ALL No(\%) } & \multicolumn{3}{|c|}{ T.ALL No(\%) } & \\
\hline & & & & Male & Female & & Male & Female & $0-14$ & $>14 \mathrm{yrs}$ & & $0-14$ & $>14 y r s$ & \\
\hline CD1a & Neg & $8 / 18(44.4)$ & & $\mathrm{Neg}$ & $\mathrm{Neg}$ & & $6 / 15(40)$ & $2 / 3(66.7)$ & $\mathrm{Neg}$ & $\mathrm{Neg}$ & & $8 / 11(72.7)$ & $\mathrm{Neg}$ & \\
\hline CD2 & $\mathrm{Neg}$ & $13 / 18(72.4)$ & & $\mathrm{Neg}$ & $\mathrm{Neg}$ & & $12 / 15(80)$ & $1 / 3(33.3)$ & $\mathrm{Neg}$ & $\mathrm{Neg}$ & & 9/11(81.8) & $4 / 7(57.1)$ & \\
\hline CD3 & Neg & 7/18(38.9) & & $\mathrm{Neg}$ & $\mathrm{Neg}$ & & $5 / 15(33.3)$ & $2 / 3(66.7)$ & $\mathrm{Neg}$ & $\mathrm{Neg}$ & & 4/11(36.4) & $3 / 7(42.8)$ & \\
\hline cCD3 & Neg & $18 / 18(100)$ & & $\mathrm{Neg}$ & $\mathrm{Neg}$ & & $15 / 15(100)$ & $3 / 3(100)$ & $\mathrm{Neg}$ & Neg & & $11 / 11(100)$ & $7 / 7(100)$ & \\
\hline CD4 & Neg & $8 / 18(44.4)$ & & $\mathrm{Neg}$ & $\mathrm{Neg}$ & & $6 / 15(40)$ & $2 / 3(66.7)$ & $\mathrm{Neg}$ & $\mathrm{Neg}$ & & $8 / 11(72.7)$ & $\mathrm{Neg}$ & \\
\hline CD8 & $\mathrm{Neg}$ & $13 / 18(72.2)$ & & $\mathrm{Neg}$ & Neg & & $10 / 15(66.6)$ & $3 / 3(100)$ & $\mathrm{Neg}$ & Neg & & 10/11(90.9) & $3 / 7(42.8)$ & $\mathbf{S}^{*}$ \\
\hline CD5 & $\mathrm{Neg}$ & 16/18(88.9) & & Neg & $\mathrm{Neg}$ & & $13 / 15(86.7)$ & $3 / 3(100)$ & Neg & Neg & & $11 / 11(100)$ & $5 / 7(71.4)$ & $\mathbf{S}^{*}$ \\
\hline CD7 & $2 / 69(2.9)$ & $18 / 18(100)$ & $S^{*}$ & $1 / 38(2.6)$ & $1 / 31(3.2)$ & & $15 / 15(100)$ & $3 / 3(100)$ & $1 / \mathbf{4 4}(2.3)$ & $1 / 25(4)$ & & $11 / 11(100)$ & $7 / 7(100)$ & \\
\hline TdT & $50 / 58(86.2)$ & $8 / 14(57.1)$ & $\mathbf{S}^{*}$ & $28 / 31(90.3)$ & $22 / 27(81.5)$ & & $6 / 12(50)$ & $2 / 3(66.7)$ & $34 / 38(89.5)$ & $16 / 20(80)$ & & $6 / 10(60)$ & $2 / 5(40)$ & \\
\hline CD4+CD8+ & 0 & 9/18(50) & & 0 & 0 & & $7 / 15(46.7)$ & $2 / 3(66.7)$ & 0 & 0 & & 9/11(81.8) & 0 & \\
\hline CD4-CD8- & 2/69(2.9) & 4/18(22.2) & $\mathbf{S}^{*}$ & 1/38(2.6) & 1/31(3.2) & & $4 / 15(26.7)$ & 0 & $1 / 44(2.3)$ & $1 / 25(4)$ & & 2/11(18.2) & 2/7(28.6) & \\
\hline CD4-CD8+ & 0 & 2/18(11.1) & & 0 & 0 & & $1 / 15(6.7)$ & 1/3(33.3) & 0 & 0 & & 0 & 2/7(28.6) & \\
\hline CD10+CD19+ & $50 / 69(72.5)$ & 0 & & 30/38(78.9) & $20 / 31(64.5)$ & & 0 & 0 & $35 / 44(79.5)$ & $15 / 25(60)$ & & 0 & 0 & \\
\hline CD33+CD19+ & $1 / 69(1.4)$ & 0 & & 0 & 1/31(3.2) & & 0 & 0 & $1 / \mathbf{4 4}(2.3)$ & 0 & & 0 & 0 & \\
\hline No Dual & $16 / 69(23.2)$ & $3 / 18(16.7)$ & & 7/38(18.4) & 9/31(29) & & $3 / 15(20)$ & 0 & $7 / 44(15.9)$ & 9/25(36) & & 0 & $3 / 7(42.8)$ & \\
\hline CD10 & $54 / 68(79.4)$ & $4 / 18(22.2)$ & $\mathbf{S}^{*}$ & $32 / 37(86.5)$ & 22/31(70.9) & & $4 / 15(26.7)$ & Neg & $38 / 43(88.4)$ & $16 / 25(64)$ & $\mathrm{S}^{*}$ & 3/11(27.3) & $1 / 7(14.3)$ & \\
\hline CD19 & 69/69(100) & $\mathrm{Neg}$ & & $38 / 38(100)$ & $31 / 31(100)$ & & $\mathrm{Neg}$ & $\mathrm{Neg}$ & $\mathbf{4 4 / 4 4 ( 1 0 0 )}$ & $25 / 25(100)$ & & $\mathrm{Neg}$ & $\mathrm{Neg}$ & \\
\hline CD20 & $30 / 69(43.5)$ & $\mathrm{Neg}$ & & 21/38(55.3) & 9/31(29) & $\mathbf{S}^{*}$ & $\mathrm{Neg}$ & Neg & $16 / 44(36.4)$ & $14 / 25(56)$ & & Neg & $\mathrm{Neg}$ & \\
\hline CD22 & $16 / 64(25)$ & $\mathrm{Neg}$ & & $10 / 35(28.6)$ & 6/29(20.7) & & $\mathrm{Neg}$ & $\mathrm{Neg}$ & $10 / 39(25.6)$ & $6 / 25(24)$ & & $\mathrm{Neg}$ & $\mathrm{Neg}$ & \\
\hline cCD22 & 22/48(45.8) & Neg & & $13 / 25(52)$ & 9/20(45) & & $\mathrm{Neg}$ & Neg & $15 / 30(50)$ & 7/19(36.8) & & $\mathrm{Neg}$ & $\mathrm{Neg}$ & \\
\hline cCD79A & $55 / 57(96.5)$ & $\mathrm{Neg}$ & & $31 / 32(96.8)$ & $24 / 25(96)$ & & $\mathrm{Neg}$ & $\mathrm{Neg}$ & $36 / 37(97.3)$ & $17 / 25(68)$ & $\mathbf{S}^{*}$ & $\mathrm{Neg}$ & $\mathrm{Neg}$ & \\
\hline CD34 & $42 / 69(60.9)$ & $5 / 18(27.8)$ & $\mathrm{S}^{*}$ & 22/38(57.9) & $20 / 31(64.5)$ & & $5 / 15(33.3)$ & $\mathrm{Neg}$ & $25 / 44(56.8)$ & $19 / 20(95)$ & $\mathbf{S}^{*}$ & $1 / 11(9.1)$ & $4 / 7(57.1)$ & $\mathbf{S}^{*}$ \\
\hline CD45(low) & $55 / 69(79.7)$ & 2/18(11.1)@ & & $27 / 38(71)$ & $28 / 31(90.3)$ & & $14 / 15(93.3)$ & $3 / 3(100)$ & $35 / 44(79.5)$ & $20 / 25(80)$ & & 10/11(90.9) & $7 / 7(100)$ & \\
\hline HLADR & 68/69(98.6) & 2/18(11.1) & $\mathbf{S}^{*}$ & $37 / 38(97.4)$ & $31 / 31(100)$ & & 2/15(13.3) & $\mathrm{Neg}$ & $44 / 44(100)$ & 24/25(96) & & $\mathrm{Neg}$ & 2/7(28.6) & \\
\hline CD13 & $4 / 69(5.8)$ & $\mathrm{Neg}$ & & 2/38(5.3) & 2/31(6.4) & & $\mathrm{Neg}$ & $\mathrm{Neg}$ & $2 / 44(4.5)$ & $2 / 25(8)$ & & $\mathrm{Neg}$ & $\mathrm{Neg}$ & \\
\hline CD33 & 2/69(2.9) & 1/18(5.6) & & 1/38(2.6) & $1 / 31(3.2)$ & & $1 / 15(6.7)$ & $\mathrm{Neg}$ & $2 / 44(4.5)$ & Neg & & Neg & $1 / 7(14.3)$ & \\
\hline CD14 & Neg & Neg & & $\mathrm{Neg}$ & Neg & & $\mathrm{Neg}$ & $\mathrm{Neg}$ & $\mathrm{Neg}$ & Neg & & $\mathrm{Neg}$ & $\mathrm{Neg}$ & \\
\hline CD117 & $1 / 68(1.47)$ & $2 / 15(13.3)$ & $S^{*}$ & $\mathrm{Neg}$ & 1/31(3.2) & & 2/13(15.4) & $\mathrm{Neg}$ & $1 / \mathbf{4 4}(2.3)$ & Neg & & Neg & $2 / 5(40)$ & \\
\hline CD64 & $\mathrm{Neg}$ & $\mathrm{Neg}$ & & Neg & Neg & & $\mathrm{Neg}$ & $\mathrm{Neg}$ & $\mathrm{Neg}$ & Neg & & $\mathrm{Neg}$ & $\mathrm{Neg}$ & \\
\hline TdT+CD34+ & $31 / 56(55.3)$ & $1 / 14(7.14)$ & $S^{*}$ & $16 / 30(53.3)$ & $15 / 26(57.7)$ & & $1 / 12(8.3)$ & 0 & 19/37(51.3) & $12 / 19(63.1)$ & $\mathbf{S}^{*}$ & 0 & $1 / 4(25)$ & \\
\hline TdT-CD34- & $6 / 56(10.7)$ & 4/14(28.6) & & $2 / 30(6.7)$ & $4 / 26(15.4)$ & & 4/12(33.3) & 0 & 3/37(8.1) & 3/19(15.8) & & $3 / 10(30)$ & $1 / 4(25)$ & \\
\hline TdT-CD34+ & 0 & $2 / 14(14.3)$ & & 0 & $\mathbf{0}$ & & 2/12(16.6) & 0 & 0 & 0 & & 1/10(10) & 1/4(25) & \\
\hline TdT+CD34- & 19/56(33.9) & 7/14(50) & & $12 / 30(40)$ & $7 / 26(26.9)$ & & $5 / 12(41.7)$ & $2 / 2(100)$ & $15 / 37(40.5)$ & $4 / 19(21)$ & & $6 / 10(60)$ & 1/4(25) & \\
\hline MPO & $\mathrm{Neg}$ & $\mathrm{Neg}$ & & $\mathrm{Neg}$ & $\mathrm{Neg}$ & & $\mathrm{Neg}$ & $\mathrm{Neg}$ & $\mathrm{Neg}$ & $\mathrm{Neg}$ & & $\mathrm{Neg}$ & $\mathrm{Neg}$ & \\
\hline
\end{tabular}


Table 2. Myeloid and T-lymphocyte antigen expression in B-cell acute lymphoblastic leukemia

\begin{tabular}{ccccccc}
\hline Antigen & All cases & Children & P value $\dagger$ & Adult & P value & P value* \\
\hline CD13 & $4 / 64(5.8 \%)$ & $2 / 44(4.5 \%)$ & 0.7 & $2 / 25(8 \%)$ & 0.7 & 0.55 \\
\hline CD33 & $2 / 69(2.9 \%)$ & $2 / 44(4.5 \%)$ & 0.6 & Negative & 0.38 & 0.27 \\
\hline CD117 & $1 / 68(1.74 \%)$ & $1 / 44(2.3 \%)$ & 0.7 & Negative & 0.54 & 0.44 \\
\hline CD14 & Negative & Negative & --- & Negative & --- & --- \\
\hline CD64 & Negative & Negative & --- & Negative & -- & -- \\
\hline MPO & Negative & Negative & --- & Negative & --- & -- \\
\hline CD7 & $2 / 69(2.9 \%)$ & $1 / 44(2.3 \%)$ & 0.8 & $1 / 25(4 \%)$ & 0.7 & 0.68 \\
\hline
\end{tabular}

$\dagger \mathrm{P}$ value significant if $\leq 0.05$ for positive aberrant marker in children versus all cases

$\ddagger$ value significant if $\leq 0.05$ for positive aberrant marker in adults versus all cases

$* P$ value significant if $\leq 0.05$ for positive aberrant marker in children versus adult patients

Table 3. Aberrant Ag expression in T cell acute lymphoblastic leukemia

\begin{tabular}{ccccccc}
\hline Antigen & All cases & Children & P-value $\dagger$ & Adult & P-value $\neq$ & P-value* \\
\hline CD10 & $4 / 18(22 / 2 \%)$ & $3 / 11(2.7 \%)$ & 0.7 & $1 / 7(14.3 \%)$ & 0.6 & 0.5 \\
\hline CD13 & Negative & Negative & --- & Negative & -- & -- \\
\hline CD33 & $1 / 18(5.6 \%)$ & Negative & 0.6 & $1 / 7(14.3 \%)$ & 0.4 & 0.19 \\
\hline CD117 & $2 / 15(13.3 \%)$ & Negative & 0.2 & $2 / 5(14 \%)$ & 0.19 & 0.02 \\
\hline HLADR & $2 / 18(11.1 \%)$ & Negative & 0.2 & $2 / 7(28.6 \%)$ & 0.2 & 0.06 \\
\hline
\end{tabular}

$\dagger \mathrm{P}$ value significant if $\leq 0.05$ for positive aberrant marker in children versus all cases

$\ddagger$ P value significant if $\leq 0.05$ for positive aberrant marker in adults versus all cases

$* \mathrm{P}$ value significant if $\leq 0.05$ for positive aberrant marker in children versus adult patients

Table 4. Aberrant $\mathrm{Ag}$ expression in acute lymphoblastic leukemia

\begin{tabular}{cccc}
\hline Antigen & T cell & B cell & P-value \\
\hline CD13 & Negative & $4 / 64(5.8 \%)$ & 0.27 \\
\hline CD33 & $1 / 18(5.6 \%)$ & $2 / 69(2.9 \%)$ & 0.5 \\
\hline CD117 & $2 / 15(13.3 \%)$ & $1 / 68(1.74 \%)$ & 0.02 \\
\hline
\end{tabular}

\section{Discussion}

In this study, we focused on the evaluation of ALL surface and cytoplasmic antigens. We reviewed the expression frequency of different markers including aberrancies in patients with ALL to set aside the prevalence of antigens and reach the minimum necessary diagnostic panels by flow cytometry in Iran. According to our findings, aberrant phenotypes including CD13, CD33, CD7, and CD117 were found in seven cases $(10.1 \%)$ of B-LBL. These phenotypes were CD10, CD117, HLA-DR, and CD33 in 7 cases (38/9\%) of T-LBL. Expression of CD117 aberrant myeloid antigen was significantly more associated with T-LBL than with B-lineage ALL $(P=0.02)$.

In our study, CD7 expression in B-LBL was 2 out of 69 cases in the precursor of B-LBL, which is about
$2.9 \%$. This is less than the study by Basturk et al. (12) that revealed 6 out of 41 cases $(14.6 \%)$ and it is higher than some other studies (16) which reported that CD7 expression was 6 out of 134 (11) and 4 out of 200 (16). Although CD7 is considered as a poor prognostic marker in these studies $(12,16)$ it is a good marker in other studies (11). Unfortunately, we did not review 6-month recurrent rates and $6^{\text {th }}$ and $12^{\text {th }}$ month survival rates.

In addition, expression rate of surface and cytoplasmic CD22 was $25 \%$ and $45.8 \%$ of cases, respectively. Basturk et al. reported cytoplasmic CD22 in 10 out of $40(25 \%)$ B-LBL cases with higher survival rate (12). Expression of CD117 as an immature myeloid lineage marker in B-LBL has been considered as aberrant marker. Seegmiller et al. (11) reported 
CD117 in 1 out of $200(0.5 \%)$ and in the study conducted in Indonesia (13), 4 out of 183 patients $(2.2 \%)$ was reported; also, Basturk et al. (12) reported 9 out of 25 cases (36\%) with poor prognosis. In our study, one out of 68 cases $(1.47 \%)$ was positive for CD117 which is closer to the first two previous studies that prognostic effect is not described in the these studies for CD117. CD19 is expressed in all of the BLBL cases. TdT was positive in $86 \%$ of cases in our study, while it was positive in $68.3 \%$ to $97.4 \%$ in another study $(12,17)$.

Our study showed that in the group of B-LBL in the male patients, CD20 antigen had a significant difference $(P=0.02)$, while CD45 low expression in female patients had a significant difference compared to the male group $(P=0.04)$. This finding suggests more mature blasts in males than females with B-LBL. In addition, CD117 was not detected in any of the male patients, while female cases were positive in $3.2 \%$.

In B-LBL patients, CD10 antigen was seen more in children $(P=0.01)$. This is according to a survey conducted by Seegmiller (11); but unlike this study, in our study, the expression of CD45 low does not show a significant difference between children and adult groups. On the other hand, the expression of CD34 in adult shows a greater incidence, while in the study by Basturk (12) CD34 expression was observed more significantly.

Since simultaneously use of flow cytometry and cytologic analysis such as fluorescence in situ hybridization (FISH) and conventional karyotyping was not possible in our center, comparing cytogenetic findings was not done between the groups. Seegmiller et al. (11) reported absence of CD10 in adults in accordance with $11 \mathrm{q} 23$ gene rearrangement; also, CD10+/CD19+ has been seen more in children and CD10-/CD19 + more in adults.

Cytoplasmic cCD79a expression in the children under 14 years old was much higher than adults. Also, TdT+/CD34+ was higher in adults than children. Therefore, it seems that in B-LBL, blast cells are more immature in adults.

Since in this study, CD7, CD13, CD33, and CD117 were the most aberrant markers expressed in B-LBL, these markers should be considered in routine diagnostic panel for leukemia, not only in initially diagnosis processes, but also after treatment and for minimal residual disease (MRD) follow-up. CD14 and CD64 were not seen in B-LBL patients. These antigens are in first place of diagnosis and they are indicator markers that show the leukemic blast cells, which their tracking is helpful in differential diagnosis of hematogone hyperplasia from MRD. While CD14 and CD64 were not seen in B-LBL patients, Seegmiller et al. (11) reported CD14 and CD64 in $1.5 \%$ and $6.5 \%$ of cases, respectively; but in another study this expression rate was $5 \%$ for CD14 (18).

In our study, the $\mathrm{CD} 7$ and $\mathrm{CcD} 3$ were positive in $100 \%$ of T-LBL cases and the frequency of CD3 and
CD5 antigens in T-LBL were $38.9 \%$ and $88.9 \%$, respectively. The frequency of $\mathrm{CD} 3, \mathrm{CD} 5$, and $\mathrm{CD} 7$ in our study was similar to a previous study (17). Our study found that CD7 is the most sensitive marker in TLBL, which is consistent with mentioned study.

Because of the low expression of surface CD3 in TLBL, which almost always occurs in the cytoplasm, surface CD3 has been seen with low expression. CD10 in $22.2 \%$ and CD19 and CD20 were not seen in any of the T-LBL cases, that is similar to the study by Salem (17) which reported that CD7 expression was in $23 \%$ in T-LBL cases with no expression for CD19 and CD20.

CD13 expression was not seen in any T-LBL cases, while CD33 and CD117 were positive in 5.6\% and $13.3 \%$ of patients, respectively. This is similar to the study by Salem (17) which did not report CD13 and CD33 expression. In addition, our study revealed HLA-DR expression in $11.1 \%$ of T-LBL cases with no report in children. But Salem reported no HLA-DR expression in T-LBL. This expression is considered $10 \%$ in the textbook of flow cytometry (15). Moreover, Seegmiller et al. reported CD13, CD33, and CD117 in $60 \%$ of cases (11).

Mazher et al. (18) reported CD13, CD33, and MPO expression in $28 \%, 28 \%$, and $14 \%$ of T-LBL cases, respectively; these percentages are different from our findings that CD13 and CD33 were observed in 0\%, $5.6 \%$, and $0 \%$ of cases, respectively. Also, in the study by Sarma (14), CD117 expression was aberrant antigen in $54 \%$ of ALL cases, which is the most common positive myeloid marker in ALL; meanwhile, our results showed that $\mathrm{CD} 117$ is the most common myeloid aberrant antigen in T-LBL (Table 3).

According to the text book of flow cytometry (15), CD117 expression as aberrant antigen was reported in $11 \%$ of $\mathrm{T}-\mathrm{LBL}$ cases that is very close to our finding (CD117=13.3\%). CD10, CD4-/CD8- expression are aberrant antigens in $24 \%$ and $43 \%$, respectively. Our study demonstrated $22.2 \%$ for both markers and CD4+/CD8+ expression was $50 \%$, but it was $39 \%$ in textbook of flow cytometry.

CD4+/CD8+ expression was not seen in adults, while CD8-/CD4- and CD34 was higher in adults than children. This also demonstrates that blast cells are more immature in adult T-LBL. CD117 as aberrant antigen in adult T-LBL showed more expression, while it was the opposite in B-LBL. TdT+/CD34+ has been observed more in adults T-LBL which shows more immature blast cells in these cases.

Therefore, the studies conducted in different areas represent different aberrant phenotype incidence in patients with ALL. This finding might be due to genetic variation in geographic distribution, if we can exclude the effects of using different reagents (14).

CD33 has also been seen in T-LBL adults, while the opposite is true in patients with B-ALL. Thus, CD14 was not observed as aberrant antigen in any of the age and genders groups in B-LBL and T-ALL. 
In patients with T-LBL, CD10, CD33, and CD117 were more expressed as aberrant antigens .In comparison, TDT+/CD34+ cases were more observed in B-LBL, and the difference was statistically significant. It seems that in T-LBL, both markers should be used as dual. TdT-/CD34- were more detected in T-LBL than B-LBL, although they were not statistically significant.

Briefly, according to the findings of this study, panel of flow cytometry markers including CD1a, CD2, CD3, CD4, CD8, CD7, TdT, CD34, CD33, and CD117 had more diagnostic value in T-LBL. In patients with B-LBL, markers such as CD10, CD19, CCD79a, CD13, CD33, CD117, TdT, and CD34 should be considered as a part of the initial diagnostic panel. In addition, considering absent expression of CD14 and CD64, it seems that it is not necessary to use them in the diagnostic panel for ALL, whereas CD33 and CD117 with higher expression in ALL; then myeloid should be considered for diagnostic panel.

The present study had several limitations. In this study, cytogenetic analysis was not done and the limited sample size in T-LBL group decreased the statistical value. In this context, studies with lager sample size and cytogenetic analysis for determining diagnostic panel markers with considering the relation between incidence of markers and treatment prognosis are suggested. Further studies with meta-analysis could show the geographical effects on phenotypic characteristic of blast cells. However, according to this study, these phenotypic analyses in each geographic region could explain that more common antigens were more common in ALL.

\section{Acknowledgements}

This study was conducted with the approval of Iranian Blood Transfusion Organization (IBTO) and utilizing protocols approved by the respective institutional review boards.

This article has been extracted from the thesis written by Dr Najme Esfandiari in the School of Medicine, Shahid Beheshti University of Medical Sciences (Registration No: $77 \mathrm{M}$ ).

All patients signed a written consent letter. The datasets generated during and/or analyzed during the current study are available upon request.

\section{Conflict of Interest}

The authors declared that there is no conflict of interest regarding the publication of this article.

\section{References}

1. Banihashem A, Ghasemi A, Tavasolian L. Association of cytogenetics and immunophenotype in prognosis of children with acute lymphoblastic leukemia: Literature Review. Reviews in clinical medicine. 2014;1(1):2-6.

2. Pui C-H, Robison LL, Look AT. Acute lymphoblastic leukaemia. The Lancet. 2008;371(9617):1030-43. [DOI:10.1016/S0140-6736(08)60457-2]

3. Mousavi SM, Pourfeizi A, Dastgiri S. Childhood cancer in Iran. Journal of pediatric hematology/oncology. 2010;32(5):376-82. [DOI:10.1097/MPH.0b013e3181e003f7] [PMID]

4. Shadi Kolahdoozan MD M, Alireza Sadjadi MD M, Radmard AR, Hooman Khademi MD M. Five common cancers in Iran. Archives of Iranian medicine. 2010;13(2):143.

5. Dastgiri S, Fozounkhah S, Shokrgozar S, Taghavinia M, Asvadi Kermani A. Incidence of Leukemia in the Northwest of Iran. Health Promot Perspect. 2011;1(1):503.

6. Alebouyeh M. Current status of pediatric oncology in Iran. Arch Iranian Med. 2003;6(3):160-2.

7. Udomsakdi-Auewarakul $\mathrm{C}$, Promsuwicha $\mathrm{O}$, Tocharoentanaphol C, Munhketvit C, Pattanapanyasat K, Issaragrisil S. Immunophenotypes and outcome of Philadelphia chromosome-positive and-negative Thai adult acute lymphoblastic leukemia. International journal of hematology. 2003;78(4):337-43. [DOI:10.1007/BF02983559] [PMID]

8. McPherson RA, Pincus MR. Henry's clinical diagnosis and management by laboratory methods. 22 ed: Elsevier Health Sciences; 2016.

9. Paredes-Aguilera R, Romero-Guzman L, Lopez-Santiago $\mathrm{N}$, Burbano-Ceron L, Monte CD, Nieto-Martinez S. Flow cytometric analysis of cell-surface and intracellular antigens in the diagnosis of acute leukemia. American journal of hematology. 2001;68(2):69-74. [DOI:10.1002/ajh.1155] [PMID]

10. Gorczyca W. Flow Cytometry in Neoplastic Hematology: Morphologic--Immunophenotypic Correlation. London: Informa UK Ltd; 2006.

11. Seegmiller AC, Kroft SH, Karandikar NJ, McKenna RW. Characterization of immunophenotypic aberrancies in 200 cases of $\mathrm{B}$ acute lymphoblastic leukemia. American journal of clinical pathology. 2009;132(6):940-9. [DOI:10.1309/AJCP8G5RMTWUEMUU] [PMID]

12. Basturk A, Akinci S, Hacibekiroglu T, Guney T, Kutlucan A, Ceran F, et al. Prognostic significance of flow cytometry findings in Turkish adult acute leukemia patients. European review for medical and pharmacological sciences. 2015;19(18):3360-6.

13. Supriyadi E, Veerman AJ, Purwanto I, Cloos J. Myeloid antigen expression in childhood acute lymphoblastic leukemia and its relevance for clinical outcome in Indonesian ALL-2006 protocol. Journal of oncology. 2012;2012. [DOI:10.1155/2012/135186] [PMID] [PMCID] 
14. Sarma A, Hazarika M, Das D, Kumar Rai A, Sharma JD, Bhuyan $\mathrm{C}$, et al. Expression of aberrant $\mathrm{CD}$ markers in acute leukemia: A study of 100 cases with immunophenotyping by multiparameter flowcytometry. Cancer Biomarkers. 2015;15(4):501-5. [DOI:10.3233/CBM-150482] [PMID]

15. Gorczyca W. Flow Cytometry in Neoplastic Hematology: Morphologic--Immunophenotypic Correlation: CRC Press; 2010. [DOI:10.3109/9781841847443] [PMCID]
16. Hussein S, Gill KZ, Sireci AN, Colovai AI, Small T, Emmons FN, et al. Aberrant T-cell antigen expression in B lymphoblastic leukaemia. British journal of haematology. 2011;155(4):449-56. [DOI:10.1111/j.13652141.2011.08870.x] [PMID]

17. Salem DA, El-Aziz SMA. Flowcytometric immunophenotypic profile of acute leukemia: mansoura experience. Indian journal of hematology and blood transfusion. 2012;28(2):89-96. [DOI:10.1007/s12288011-0110-2] [PMID] [PMCID]

18. Chughtai O, Chughtai A. Aberrant Expression of $\mathrm{CD}$ Markers in Acute Leukemia. Ann Pak Inst Med Sci. 2013;9(2):99-102.

\section{How to Cite This Article}

Rezaei, M., Refoua, S., Esfandiari, N. Characterization of Immunophenotypic Aberrancies in Adult and Childhood Acute Lymphoblastic Leukemia: Lessons from Regional Variation. Iranian Journal of Pathology, 2020; 15(1):1-7. doi: 10.30699/ijp.2019.93974.1926 